\title{
Plant molecular breeding: objectives, safety issues and prospects
}

\author{
FRANCISCO GARCIA-OLMEDO \\ Departamento de Biotecnología de la Universidad Politécnica de Madrid E. \\ T. S. Ingenieros Agrónomos. 28040 Madrid, Spain. E-mail: \\ olmedo@bit.etsia.upm.es
}

Plant molecular breeding represents a new technology that adds to, rather than substitutes for, traditional breeding practice, and shares with it the same long-standing objectives: higher yield, better products, better-adapted plants. Additionally, it allows the consideration of completely new applications: pharmaceuticals, industrial products and bioremediation processes. Safety issues and the socio-economic prospects of this new technology are discussed.

\section{Novel versus traditional technologies}

Crop species ceased to be 'natural' - that is, lost their ability to survive by themselves in Nature - in the process of acquiring their main agronomic traits. Modern molecular techniques are clarifying how drastic were the genetic changes introduced during domestication, as well as during the slow breeding process that took place in subsequent centuries. Genetic engineering methods are now being extensively applied to the improvement of crop plants; however, the new technology will not replace the well-established plant breeding methods that have been developed over the past ten millennia.

Plant genomes, which contain 25 000-30000 genes, have undergone numerous changes in the course of the improvement of both multigenic and oligogenic agronomic traits. Classical breeding techniques are still the most effective approach when dealing with traits controlled by multiple genes distributed over the genome, especially with the aid of molecular markers, whereas genetic engineering is to be preferred in the manipulation of traits that depend on one or a few genes. Novel methods add a new dimension to traditional plant breeding practice with respect to its long-term objectives, such as yield and quality 
improvement. Additionally, recombinant DNA technology is opening the way to entirely new objectives that were previously out of reach.

\section{Current agricultural challenges}

Since middle of the 20th century, the attainment of higher yields and the development of cleaner farming practices have been the two main challenges of agriculture. Owing to demographic growth and a rate of soil decay that is difficult to compensate by new soil development, available soil per capita has steadily decreased in the past decades, from around 0.5 hectares to half that figure at present. In the next 20 years it will be further reduced to $1000-1500$ square metres per capita. So it must be concluded that, at a global scale, there is no option but to increase yield ( $\mathrm{Tm} / \mathrm{ha}$ ) of all the main crops. This objective has a special significance in relation to biodiversity because, by increasing yields, it will be possible to avoid further invasion of natural habitats.

Agricultural practice has been extremely deleterious to the environment since its invention, ten millennia ago, to the present time. This negative impact has increased concomitantly with the growth of world population. Although the production of a ton of food with a modem cultivar of wheat or com requires less soil, less energy and smaller amounts of fertilizers and phyto-chemicals than did similar food under cultivation 30 years ago, the doubling of the world population during this period has more than offset the technological progress. The negative impact of agricultural production has reached an intolerable magnitude, both to the environment, in general, and to biodiversity, in particular.

Yields of the staple grain crops have been steadily increasing over the past decades but, in recent years, this trend is clearly levelling out. Even if we extrapolate to the next 30 years the successful increases of the past, the projection shows grain deficits in all areas of the world with the exception of Europe and the North-American continent.'

\section{Old and novel objectives}

Traditional plant breeding objectives have included the modification of traits that are directly related to increased yield, as well as with the improvement of quality and other traits that are of interest in the context of sectorial demands. Most of the transgenic crop plants that have been approved for cultivation so far do address the above-stated challenges of higher-yielding, cleaner agriculture. In effect, pest and disease resistant cultivars have the potential to increase yields, by reducing losses, and to allow for cleaner agricultural practice, by reducing the requirement for phyto-chemicals. For example, insect resistance in half of the cotton cultivated in Spain would reduce pesticide use by almost 1000 tons. Other applications also 
have the potential to reduce environmental impacts. Thus, herbicide-resistant cultivars allow minimum-tillage, a practice that drastically reduces erosion and is clearly beneficial to the biodiversity of soil biota in the farming plot. In addition, according to a survey carried out by the American Soybean Association, the change to herbicide-resistant plants and to reduced-tillage practices for this crop in the US has led to an estimated reduction in energy consumption that represents 886 million litres of fuel per year. Whether or not each transgenic application fulfils specific expectations requires a case-by-case discussion.

Of course, a number of innovations do not respond to the main agricultural challenges and address sectorial demands, from alterations of post-harvest properties for food processing, such as delayed-ripening tomatoes, to those that meet certain nutritive requirements, as is the case of a rice cultivar with enhanced content of provitamin $A$ and iron.

Often overlooked is the fact that the new techniques allow the engineering of transgenic plants whose sole purpose is to serve in the screening of a new generation of phyto-chemicals with environment-compatible properties, i.e. higher specific activity (from $\mathrm{kg} / \mathrm{ha}$ to $\mathrm{g} / \mathrm{ha}$ ), greater selectivity (so that they ideally affect only the organisms to be controlled) and higher biodegradability (so that they do not accumulate in the environment).

Besides converging with classical methods in solving long-standing problems, genetic engineering has the potential to introduce completely new properties into cultivated plants. These innovations include plants that produce molecules of pharmaceutical interest, such as vaccines or peptide hormones, or of industrial interest, such as new oils or biodegradable plastics, and plants that are more effective in the remediation of contaminated soils or as pollution monitors.

The relevance of each application will have to be judged on the basis of its individual merits. Transgenic technology is here to stay. Over $\mathbf{4 5}$ million ha of transgenic crops are already cultivated in more than a dozen countries. There should not be any doubt that, generically speaking, this technology offers great promise as an ingredient for possible solutions to the problem of how to conciliate the production of food for an increasing population with the goal of conserving the environment and promoting biodiversity.

\section{Safety issues}

\section{General considerations}

As with any other technology, there is no simple general assessment concerning the safety of genetic engineering. ${ }^{2-6}$ This issue has to be treated on a case-by-case basis as, indeed, transgenic crops are approved for commercial use through an 
application-by-application procedure. Zero risk is a myth, an ideal that is not met by any human endeavour. As a negative scientific proposition it is not even susceptible of being tested experimentally.

Each proposal of transgenic application has to be evaluated on the basis of benefits-versus-risk and risk-versus-risk considerations. The implementation of an approved innovation can be closely monitored, so that the approval can be cancelled if the expectations derived from the initial assessment are off target.

The concept of substantial equivalence, which implies a direct comparison of the proposed innovation with that currently in use, offers a rational approach to the problem. Both intended and unintended effects should be investigated and assessed. Intended effects can be generally evaluated by well-established standard protocols whose application is not restricted to transgenic products. Additionally, new tools, high throughput genomic, proteomic and metabolomic methods have been developed that allow the investigation of unintended effects, both predictable and unpredictable, of genetic alterations, independently of the method used to achieve these alterations - classical breeding or genetic engineering. Indeed, risk issues are not conceptually different for traditionally bred or for genetically engineered cultivars.

\section{Safety for humans}

Safety considerations concerning the possible effects of transgenic products on humans should address possible toxic effects and potential allergenic problems. Standard chronic toxicity tests can be performed with the transgenic material, using the isogenic non-transgenic material as a control. Additionally, the toxicity of new products differentially present in the transgenic line, whether intended or unintended, can be individually assessed if required. Precedents in the literature are of great help in this type of assessment.

Allergy problems affect an increasing number of urban dwellers for reasons that are not well known. A considerable number of items from our daily life - from rubber gloves to peanuts - can cause allergic reactions in sensitive individuals. Molecules (allergens) and parts of them (epitopes) responsible for this phenomenon have been identified in many cases, a circumstance that facilitates their analytical monitoring in all kinds of materials, while in other cases, only the overall allergenicity of a particular material is known. Under the current legal framework, no gene encoding a known allergen may be expressed transgenically for commercial use and no gene from a particularly allergenic species may be transferred, unless there is evidence that the gene product is not responsible for the observed allergy. On the other hand, the potential use of transgenic technology to eliminate allergens in plants is frequently overlooked. 


\section{Gene fluxes}

Once a gene has been transferred to a particular species, it becomes an integral part of its genetic make-up and will have the same fate as the remaining thousands of genes that are part of its genome. Inter-specific genetic fluxes occur in nature to a very limited extent and are subject to barriers that are breached with a different probability in each case. A pollen grain must fly a certain distance, find an appropriate, mature recipient, pollinate, yield a viable seed, capable of developing into a non-sterile mature plant and, finally, the progeny of this plant must be viable. For certain transformation events, accidental gene transfer to other species is highly unlikely, whereas for others there is a certain probability of occurrence. These cases are susceptible to experimental evaluation. There is no danger of the transfer of plant genes into the genomes of humans or other animals. After all, humans and other animal species have daily consumed thousands of different such genes and no evidence has been found in animal genomes of this type of horizontal transfer. Also unlikely is that trans-genes will modify gut micro-flora, as there is no precedent in the literature of such an event and no evidence of it has been found in specific experiments. Nevertheless, European regulations call for phasing out antibiotic resistance genes in transgenic plants.

The transfer of genes from the cultivated species to related wild species in the same habitat occurs with a probability that is different for each species/habitat combination. Whether the species is self-fertilized or cross-fertilised, and whether or not there are closely related wild species in the vicinity of the cultivated one, determine the relevance of this issue in the approval procedure. Thus, maize is quite unproblematic from this point of view, whereas alfalfa would have to be more closely watched, and canola would represent an intermediate case. Several million hectares of transgenic canola are currently cultivated in the North-American continent, after an initial approval, and the whole operation is under close monitoring. The results of these observations will be decisive with respect to the future of this transgenic crop.

Of course, gene transfer to non-transgenic cultivars of the same species only requires appropriate synchrony and is limited by distance. This transfer is neither a health risk nor an environmental problem, but it is a commercial requirement. It is managed by planting a number of rows of non-transgenic material around the transgenic field, by keeping regulation distances between transgenic and non-transgenic plots and by monitoring adventitious events by highly sensitive analytical methods.

\section{Environmental safety}

In addition to the above-discussed problems posed by possible gene fluxes, the 
environmental safety of transgenic crops involves two main aspects that, although also relevant for classically bred cultivars, require special evaluation in genetically engineered crops. These are the possible effects on non-target organisms and the possible induction of resistance in the target organism. There are also other specialized issues that affect specific applications that need to be considered case by case.

When genetic resistance to a particular target organism (bacteria, fungi, insects and others) is introduced into a given cultivar, possible short and long-term effects on non-target organisms have to be evaluated. This evaluation has to consider current practice as a control. Thus, the effect of the introduction of BT cotton on beneficial insects has to be compared with the effect of the repeated insecticidal treatments that are included in the state-of-the-art cultivation of non-transgenic cultivars. In this context, transformation events that have been approved so far for plant protection seem to be more environmentally friendly than current technology.

Induction of resistance in the target organism is to be expected sooner or later in all cases of biological control experimented to date, whether insecticides, antibiotics or other agents are involved. This induction can be accelerated by malpractice and delayed by proper management. Contrary to popular belief, the possible induction of resistance should not prevent the application while it is useful. In the case of transgenic plants that are resistant to insects, microbial diseases or weeds, the implementation of proper management techniques, such as refuges, adequate doses and crop rotation, should be in the interest of all parties involved - farmers, authorities and companies.

\section{Socio-economic and ethical prospects}

As already stated, modern molecular breeding has a great potential in relation to the attainment of an agricultural practice that is more productive and environmentally friendly. As food demand increases, a more productive agriculture should permit a saving of wild habitats. Although safety problems are not easy to manage, this technology is susceptible for use under reasonable safety rules and is not more dangerous a priori than previous practices. This fact should not prevent a close scrutiny of the new methods because of their novelty.

Excessive consideration of the potential risks to human health and the environment should not blind us to the real socio-economic issues raised by the new technology, ${ }^{4,78}$ As it has been developed to a significant extent by private capital or by public research funds aimed at supporting industrial activity, intellectual property protection (patents and others) has been at the forefront of discussions concerning this biological revolution. Patents are no doubt necessary 
ingredients of progress. They are the most transparent system of protecting research investment, as the main alternative is industrial secrecy. However, the concentration in a few hands of most of the relevant intellectual property concerning a particular crop should be vigorously avoided.

A related socio-economic issue of great relevance also stems from the private nature of this technological innovation, in contrast with the genetic improvements achieved during the second half of the 20th century. While it is normal that companies should seek to profit out of their investments, this fact may exclude developing countries from the benefits of the new technology, despite its great potential in the solution of problems that are common to all types of countries and, more importantly, to the solution of problems that are specific to the more needy sectors of humanity. Thus, it is imperative that new mechanisms and rules should be considered to solve this question.

Apart from the ethical implications of those issues related to the possible benefits for developing countries, only general ethical principles - no different from those relevant to other industries, such as the automotive or the firearm technologies - are involved.

\section{References}

1. T. Dyson (1999) World food trends and prospects to 2025. Proc. Natl. Acad. Sci., 96, 5929-5936.

2. EMBO's Statement on Genetically Modified Organisms and the Public (18 January 2000).

3. FAO/WHO (2000) Safety aspects of genetically modified foods of plant origin. Report (June 2000)

4. Royal Society of London; National Academy of Science USA; Brazilian Academy of Science; Chinese Academy of Science; Indian National Academy of Science; Mexican Academy of Science; Third World Academy of Science (2000) Transgenic plants and world agriculture. National Academy Press Report 1-40 (July 2000)

5. Royal Society of London (1998) Genetically modified plants for food use. Statement (September 1998)

6. C. Somerville (2000) The genetically modified organism conflict. Plant Physiology, 123, 1201-1202.

7. R. W. Herdt (1998) Assisting developing countries toward food self-reliance Proc. Natl. Acad. Sci., 95, 1989-1992.

8. M. J. Chrispeels (2000) Biotechnology and the poor. Plant Physiology, $124,3-6$.

\section{About the Author}

Francisco García-Olmedo is Professor of Biochemistry and Molecular Biology at the ETS Ingenieros Agrónomos of the Universidad Politécnica de Madrid. His 
main contributions have been in the discovery of plant antibiotic peptides active in plant defence. His recent books include 'La tercera revolución verde' (in Spanish, 1998; in Italian, 2000) and 'Entre el Placer y la Necesidad. Claves para una dieta inteligente' (2001). 